\title{
Obesidade: principal fator de risco para hipertensão arterial sistêmica em adolescentes brasileiros participantes de um estudo de coorte
}

\author{
Obesity: main risk factor for systemic arterial hypertension \\ in Brazilian adolescents from a cohort study
}

Naiara Ferraz Moreira', Ana Paula Muraro', Flávia dos Santos Barbosa Brito², Regina Maria Veras Gonçalves-Silva', Rosely Sichieri' ${ }^{3}$, Márcia Gonçalves Ferreira'

' Departamento de Alimentos e Nutrição, Universidade Federal de Mato Grosso (UFMT), Cuiabá, MT, Brasil ${ }^{2}$ Departamento de Nutrição Social, Instituto de Nutrição, Universidade do Estado do Rio de Janeiro (UERJ), Rio de Janeiro, RJ, Brasil ${ }^{3}$ Departamento de Epidemiologia, Instituto de Medicina Social, UERJ, Rio de Janeiro, RJ, Brasil
Correspondência para: Naiara Ferraz Moreira (A/C Márcia Gonçalves Ferreira) Av. Marechal Deodoro, 829, ap. 1202 78005-100 - Cuiabá, MT, Brasil naiaraferraz@ymail.com

Recebido em 14/Set/2012 Aceito em 11/Mar/2013

\section{RESUMO}

Objetivo: Identificar a prevalência e os fatores associados à hipertensão arterial sistêmica (HAS) em adolescentes de uma coorte. Sujeitos e métodos: Estudo transversal com 1.716 adolescentes, de 10 a 16 anos, participantes de um estudo de coorte. Os dados sociodemográficos, econômicos e de estilo de vida foram obtidos por meio de entrevista e os dados referentes ao nascimento e à infância foram obtidos na linha de base. Foram considerados hipertensos os adolescentes que apresentaram pressão arterial sistólica e/ou diastólica acima do percentil 95, segundo a classificação preconizada pelo Second Task Force High Blood Pressure in Children and Adolescents. 0 estado nutricional foi diagnosticado pelo índice de massa corporal (IMC), segundo o escore z. O perímetro da cintura foi medido ao nível da cintura natural. A associação da HAS com as variáveis explicativas foi estimada por regressão logística. Resultados: Os entrevistados correspondem a 71,4\% dos avaliados na linha de base, sendo $50,7 \%$ do sexo masculino. A prevalência global de HAS foi de $11,7 \%$. Na análise ajustada para idade, sexo e cor de pele, a hipertensão arterial associou-se à obesidade $[O R=2,27$; IC $(95 \%)=1,64-3,14]$, mas não ao perímetro da cintura, que, após ajuste para o IMC, perdeu a associação. Os eventos precoces na vida não foram associados à HAS na adolescência. Conclusão: Os resultados enfatizam a obesidade como principal fator associado à HAS em adolescentes. Arq Bras Endocrinol Metab. 2013;57(7):520-6

\section{Descritores}

Adolescentes; hipertensão; fatores de risco; obesidade

\begin{abstract}
Objective: To identify the prevalence and factors associated with hypertension in adolescents. Subjects and methods: Cross-sectional study of 1,716 adolescents of a cohort study, aged from 10 to 16 years old sociodemographic, economic, and lifestyle characteristics were obtained from an interview, and birth weight from hospital records. Hypertension was defined as systolic or diastolic blood pressure above the $95^{\text {th }}$ percentile according to the classification recommended by the Second Task Force High Blood Pressure in Children and Adolescents. Nutritional status was diagnosed by body mass index (BMI), according to the z score from curves published by the World Health Organization. Waist circumference was measured at the natural waist. The association between hypertension and the explanatory variables was measured using logistic regression. Results: The adolescents interviewed represent $71.4 \%$ of the baseline, and $50.7 \%$ of them were males. The prevalence of hypertension was $11.7 \%$. In the multivariate analysis, after adjustment for age sex and skin color, hypertension was associated with obesity [OR $=2.27,(95 \%) \mathrm{Cl}=1.64$ to 3.14 ] but not associated with waist circumference after adjusting for BMI. Early life factors were not associated with hypertension in adolescence. Conclusion: The results show an association between obesity and hypertension among adolescents. Arq Bras Endocrinol Metab. 2013;57(7):520-6
\end{abstract}

Keywords

Adolescents; hypertension; risk factors; obesity 


\section{INTRODUÇÃO}

A hipertensão arterial sistêmica (HAS) é uma das mais importantes causas evitáveis de morte prematura (1). Segundo a Organização Mundial da Saúde (OMS), existem cerca de 800 milhões de pessoas com pressão arterial (PA) elevada em todo o mundo (1), causando mais de 7 milhões de mortes por ano (2).

A HAS é um dos cinco fatores de risco para o desenvolvimento de um terço de todas as doenças cardiovasculares (DCV); entre os outros fatores estão: o tabagismo, o consumo de álcool, a hipercolesterolemia e a obesidade (1). Se esses fatores fossem eliminados, pelo menos $80 \%$ de todas as DCV, dos acidentes vasculares encefálicos e do diabetes do tipo 2 poderiam ser evitados e mais de $40 \%$ dos cânceres poderiam ser prevenidos (3). Segundo estimativas da OMS, as mortes causadas por DCV serão responsáveis por 31,5\% do total de óbitos em 2020 e por $32,5 \%$ em 2030, com destaque para o aumento em países em desenvolvimento (1).

Embora a HAS seja mais frequente em adultos, a prevalência entre crianças e adolescentes está aumentando (4), porém esses valores, de forma global, não são conhecidos devido, principalmente, às diferenças na definição de PA elevada e à metodologia de medição da pressão arterial $(4,5)$. A HAS em idades tenras é frequentemente assintomática e facilmente despercebida, até mesmo por profissionais de saúde (4), por isso se deve enfatizar a importância dos cuidados em âmbito preventivo e curativo entre crianças e adolescentes.

Segundo recentes revisões da literatura, alguns fatores de risco associados à HAS em crianças e adolescentes são: excesso de peso, resistência à insulina, dislipidemias, distúrbios do sono, fatores relacionados ao estilo de vida como o sedentarismo e a alimentação (4-7), além de eventos precoces na vida como o baixo peso ao nascer $(4,8)$.

Considerando a crescente prevalência da HAS entre adolescentes, os fatores diversos que influenciam essa elevação e as consequências dos níveis tensionais elevados em curto prazo e em longo prazo, o objetivo do presente estudo foi identificar a prevalência e os fatores associados à HAS em adolescentes.

\section{SUJEITOS E MÉTODOS}

Trata-se de um estudo transversal, cujos dados foram obtidos de um estudo de coorte, realizado com adolescentes, de ambos os sexos, com idade entre 10 e 16 anos, matriculados em escolas públicas e privadas de en- sino fundamental e médio. Essa população participou da pesquisa: "Prevalência de tabagismo passivo e suas repercussões sobre a saúde de menores de cinco anos" na cidade de Cuiabá-MT.

$\mathrm{O}$ estudo de base foi realizado no período de maio de 1999 a janeiro de 2000, na cidade de Cuiabá-MT. A coleta de dados ocorreu em 10 postos de saúde sorteados entre os 38 existentes na cidade. $\mathrm{O}$ sorteio dos participantes foi realizado nessas unidades de saúde, totalizando 2.405 crianças, das quais 376 foram avaliadas no estudo piloto, o qual foi incluído na amostra por ter sido realizado no mesmo ano, adotando a mesma metodologia para a obtenção das informações coletadas. O plano amostral detalhado utilizado neste estudo foi descrito por Gonçalves-Silva e cols. (9).

Com base nos dados dos inquéritos de 1999/2000, foi possível identificar o nome da criança, a data de seu nascimento e o nome da mãe, propiciando a localização do adolescente nas escolas públicas e privadas do país e também no endereço domiciliar, segundo informações existentes no censo escolar (Educacenso) dos anos de 2009 a 2011. Esse censo é um levantamento de dados estatístico-educacionais de âmbito nacional, realizado todos os anos e coordenado pelo Instituto Nacional de Estudos e Pesquisas Educacionais Anísio Teixeira (INEP), com o apoio das Secretarias Estaduais de Educação. Os óbitos foram identificados por meio do Sistema de Informação sobre Mortalidade (SIM) da Secretaria de Estado da Saúde de Mato Grosso.

Dos 2.405 adolescentes pesquisados em 1999/2000, foram localizados, por meio do censo escolar, 2.088 $(86,8 \%)$. Desses, foram entrevistados $71,4 \%$ (1.716) no período de 2009 a 2011 . Foram avaliados $92,1 \%$ (1.605) dos adolescentes localizados em Cuiabá e Várzea Grande (cidade integrada geográfica e economicamente à Cuiabá). Foram também coletados dados de 74 estudantes em 17 cidades do interior do estado de Mato Grosso (representando 60\% dos 123 localizados nessas cidades). Em cinco capitais federativas visitadas (Brasília, Goiânia, Rio de Janeiro, São Paulo e Campo Grande) foram localizados 56 adolescentes, sendo entrevistados 37 (66\%). Mais detalhes sobre a busca e a coleta de dados foram descritos por Gonçalves-Silva e cols. (10).

Cinco adolescentes foram a óbito, sendo esses óbitos confirmados pelo SIM. Foram excluídos 11 adolescentes $(0,5 \%)$ que apresentavam condições de saúde que os incapacitavam para a entrevista. Entre as perdas, 70 estudantes $(2,9 \%)$ não foram autorizados pelos responsáveis a participar do estudo e cinco $(0,2 \%)$ se recusaram 
a participar. Nas análises que levam em consideração a HAS, foram excluídos 24 adolescentes por não conterem informações referentes aos valores da PA devido a problemas com a aferição da medida $(\mathrm{n}=1.692)$.

Para a coleta de dados utilizou-se um questionário com informações sociodemográficas, econômicas e de hábitos de vida. Esse questionário também possuía uma ficha onde foram anotados os dados relativos ao peso, estatura, perímetro da cintura e medidas da PA. O questionário foi previamente testado com 114 adolescentes sorteados, em uma escola pública e uma particular no município de Cuiabá, com o objetivo de corrigir possíveis falhas e padronizar o instrumento para coleta de dados.

$\mathrm{O}$ peso foi medido utilizando-se analisador de composição corporal, da marca Tanita (modelo UM-080), com variação de $0,1 \mathrm{~kg}$ e capacidade de $150 \mathrm{~kg}$. Para a mensuração da estatura foi utilizado estadiômetro portátil da marca Sanny, com variação de $1 \mathrm{~mm}$ e extensão de até $210 \mathrm{~cm}$. Essas medidas foram realizadas com o adolescente descalço, usando uniforme escolar e em posição ortostática, com a cabeça posicionada, respeitando-se o plano de Frankfurt. Foram realizadas duas mensurações de estatura, admitindo-se variação máxima de $0,5 \mathrm{~cm}$ entre elas. A média das duas medidas foi utilizada nas análises.

Para medida do perímetro da cintura utilizou-se fita métrica flexível e inextensível de $200 \mathrm{~cm}$ de comprimento e variação de $1 \mathrm{~mm}$. A medida foi realizada em duplicata, na altura da menor circunferência do tronco, ao nível da cintura natural, admitindo-se variação máxima de $1 \mathrm{~cm}$ entre as duas medidas. A aferição foi feita ao final da expiração, com o indivíduo em pé, em posição ereta, abdome relaxado, braços ao lado do corpo e os pés juntos.

A PA foi avaliada por meio de monitor automático Omron HEM-742 INT, validado para adolescentes (11). Utilizaram-se dois tamanhos de bolsas de borracha - adulto e infantil -, adequadas à circunferência do braço, para conferir melhor precisão na aferição dos níveis tensionais dos adolescentes. Foram realizadas três medidas da PA, com um intervalo mínimo de 1 minuto entre elas, sendo a média das duas últimas considerada a PA do adolescente. As medidas da pressão sistólica e/ ou diastólica que apresentaram diferença maior que 4 $\mathrm{mmHg}$ foram refeitas (12).

$\mathrm{O}$ estado nutricional dos adolescentes foi avaliado segundo sexo e idade, utilizando-se como indicador o índice de massa corporal $\left(\mathrm{IMC}=\mathrm{kg} / \mathrm{m}^{2}\right)$. Para a classificação do estado nutricional, foram adotadas as reco- mendações da OMS (13). Foram classificados como baixo peso os adolescentes que apresentaram IMC/idade $<-2$ escores-z; eutróficos, os que apresentaram IMC/ idade $\geq-2$ e $\leq+1$ escore-z; com sobrepeso, aqueles com IMC/idade $>+1$ escore-z e $\leq+2$ escores- $z$; e obesos, os com IMC/idade $>+2$ escore-z. O estado nutricional do adolescente foi categorizado em: sem excesso de peso (baixo peso e eutróficos), sobrepeso e obesidade.

A classificação da HAS foi baseada nos critérios de normalidade do Second Task Force, que, segundo as tabelas de referência por sexo, idade e estatura, define como: "normal" - níveis de pressão arterial sistólica e/ ou diastólica abaixo do percentil 90; "pré-hipertensão" - níveis entre os percentis 90 e 95; e "hipertensão" níveis acima do percentil 95 (6). Para as análises, os adolescentes foram classificados como hipertensos e não hipertensos.

Entre as variáveis sociodemográficas, foi considerada a cor autoatribuída, classificada neste estudo em duas categorias, não preta e preta. Para determinar a classificação econômica das famílias, utilizaram-se os critérios preconizados pela ABEP (Associação Brasileira de Empresas de Pesquisa) (14). O nível de atividade física dos adolescentes foi avaliado por meio do questionário da Pesquisa Nacional de Saúde do Escolar (PeNSE) (15). Para a análise dos dados, procurou-se identificar o tempo de prática de atividade física, que permitiu a categorização dos indivíduos nos seguintes grupos: inativos; insuficientemente ativos (os que praticaram atividade física de 1 a 299 minutos por semana); e ativos (os que praticaram 300 minutos ou mais de atividade física por semana), segundo o Instituto Brasileiro de Geografia e Estatística (IBGE) (15).

Em relação aos comportamentos sedentários (horas assistindo TV; uso de computador/games), os alunos responderam a duas questões objetivas: (a) Em um dia de semana comum, quantas horas você assiste TV? e (b) Em um dia de semana comum, quantas horas você usa o computador e/ou videogame? Determinou- se como comportamento sedentário o uso de TV e/ou computador/games por um tempo igual ou superior a 2 horas por dia (16).

As informações referentes ao nascimento da criança, prematuridade ou não, peso e comprimento ao nascer foram obtidas na realização do primeiro inquérito (estudo de base) por meio das informações contidas no cartão de vacinação da criança ou no registro hospitalar. Para avaliação antropométrica na linha de base, foram medidos o peso e o comprimento ou estatura, sendo os procedimentos adotados descritos por Gonçalves-Silva e cols. (9). 
Para a descrição dos dados, apresentam-se a prevalência e a razão de prevalência da HAS, segundo as variáveis estudadas. O teste do qui-quadrado foi utilizado para testar a diferença entre as proporções e a linearidade delas em relação à HAS. Em seguida, procedeu-se à análise de regressão logística para verificar a associação entre a variável dependente (HAS) e as independentes. A inclusão das variáveis no modelo de regressão logística ocorreu de acordo com a significância das associações observadas na análise bivariada, ajustando-se para potenciais fatores de confusão. Em todas as análises foi utilizado o nível de significância de 5\%. As análises foram realizadas pelo software Statistical Package for the Social Sciences (SPSS) 19.0 para Windows (SPSS Inc., Chicago, IL, EUA).

O projeto foi aprovado pelo Comitê de Ética do Hospital Universitário Júlio Muller, da Universidade Federal de Mato Grosso (Protocolo ${ }^{\circ}$ 651/CEP-HU$\mathrm{JM} / 2009$ ). Os pais ou responsáveis que permitiram a participação do adolescente na pesquisa assinaram o Termo de Consentimento Livre e Esclarecido antes da coleta de dados.

\section{RESULTADOS}

A média de idade dos adolescentes foi de 11,7 anos (DP $=1,5$ ). Os meninos totalizaram $50,7 \%$ das observações. A amostra foi constituída principalmente por estudantes da rede pública $(79,1 \%)$, de cor parda $(58,6 \%)$, e $55,9 \%$ pertenciam à classe econômica $\mathrm{C}$ (Tabela 1 ).

A prevalência global de HAS estimada foi de $11,7 \%$, sendo de $12,6 \%$ para o sexo masculino e de $10,8 \%$ para o feminino. Na infância, a prevalência de obesidade nessa coorte era de $4,9 \%$, havendo um incremento de $89,7 \%$ até a adolescência. O excesso de peso (sobrepeso e obesidade) aumentou em $34,5 \%$ nesse período. $\mathrm{Na}$ análise bivariada, o estado nutricional e o perímetro da cintura mostraram associação direta e linear com a prevalência de HAS. As demais variáveis analisadas não mostraram associação com a HAS (Tabela 2).

$\mathrm{Na}$ análise multivariada, verificou-se que a obesidade apresentou associação positiva com a HAS, independentemente dos demais fatores. Admitindo-se o modelo ajustado por sexo, idade e cor da pele, a obesidade aumentou em 1,27 vez a chance de o adolescente apresentar HAS (Tabela 3). Já o perímetro da cintura, quando adicionalmente ajustado pelo IMC, perdeu o efeito sobre a HAS. Observou-se neste estudo alta correlação entre essas duas variáveis antropométricas $(\mathrm{r}=0,88)$.
Tabela 1. Características sociodemográficas, econômicas, de estilo de vida e estado nutricional de adolescentes, 2011 ( $n=1.716)$

\begin{tabular}{|c|c|c|}
\hline & N & $\%$ \\
\hline \multicolumn{3}{|l|}{ Sexo } \\
\hline Masculino & 870 & 50,7 \\
\hline Feminino & 846 & 49,3 \\
\hline \multicolumn{3}{|l|}{ Idade } \\
\hline 10 & 409 & 23,8 \\
\hline 11 & 551 & 32,1 \\
\hline 12 & 322 & 18,8 \\
\hline 13 & 183 & 10,7 \\
\hline 14 ou mais & 251 & 14,6 \\
\hline \multicolumn{3}{|l|}{ Tipo de escola ${ }^{\mathrm{a}}$} \\
\hline Pública & 1.357 & 79,1 \\
\hline Particular & 358 & 20,9 \\
\hline \multicolumn{3}{|l|}{ Período escolar ${ }^{\mathrm{a}}$} \\
\hline Matutino & 890 & 51,9 \\
\hline Vespertino & 815 & 47,5 \\
\hline Noturno & 6 & 0,4 \\
\hline Integral & 4 & 0,2 \\
\hline \multicolumn{3}{|l|}{ Cor/Raça } \\
\hline Preta & 259 & 15,1 \\
\hline Branca & 371 & 21,6 \\
\hline Amarela & 36 & 2,1 \\
\hline Parda & 1.005 & 58,6 \\
\hline Indígena & 45 & 2,6 \\
\hline \multicolumn{3}{|l|}{ Classe econômicab } \\
\hline A & 86 & 5,0 \\
\hline B & 603 & 35,1 \\
\hline C & 959 & 55,9 \\
\hline De E & 68 & 4,0 \\
\hline \multicolumn{3}{|l|}{ Prática de atividade física ${ }^{c}$} \\
\hline Ativo & 864 & 50,3 \\
\hline Insuficientemente ativo & 811 & 47,3 \\
\hline Inativo & 41 & 2,4 \\
\hline \multicolumn{3}{|c|}{ Tempo gasto com atividades sedentárias ${ }^{d}$} \\
\hline$<2$ horas/dia & 312 & 18,2 \\
\hline 2-3 horas/dia & 641 & 37,4 \\
\hline$\geq 4$ horas/dia & 763 & 44,5 \\
\hline \multicolumn{3}{|l|}{ Estado nutricional } \\
\hline Baixo peso & 56 & 3,3 \\
\hline Eutrofia & 1.185 & 69,1 \\
\hline Sobrepeso & 315 & 18,4 \\
\hline Obesidade & 160 & 9,3 \\
\hline
\end{tabular}

a Sem informação para um adolescente; ' ${ }^{\circ}$ Classificação econômica ABEP, 2008; ' Classificação adotada na PeNSE - IBGE, 2009; ' ${ }^{\mathrm{C}}$ Classificação segundo Currie e cols. (2008). 
Tabela 2. Prevalência de hipertensão arterial sistêmica (\%), razão de prevalência (RP) e intervalo de confiança de 95\% (IC 5\%), segundo características dos adolescentes, 2011

\begin{tabular}{|c|c|c|c|c|c|}
\hline & $\mathbf{N}$ & $\%$ & $\mathbf{R P}$ & IC (95\%) & p-valor ${ }^{d}$ \\
\hline \multicolumn{6}{|l|}{ Idade } \\
\hline 10 & 401 & 12,2 & 1,00 & & \multirow{5}{*}{0,31} \\
\hline 11 & 543 & 11,6 & 0,95 & 0,$67 ; 1,35$ & \\
\hline 12 & 319 & 7,8 & 0,64 & 0,$41 ; 1,01$ & \\
\hline 13 & 181 & 15,5 & 1,27 & 0,$82 ; 1,95$ & \\
\hline$\geq 14$ & 248 & 14,5 & 1,20 & 0,$80 ; 1,78$ & \\
\hline \multicolumn{6}{|l|}{ Sexo } \\
\hline Feminino & 831 & 11,0 & 1,00 & & \\
\hline Masculino & 861 & 12,8 & 1,17 & 0,$90 ; 1,60$ & \\
\hline \multicolumn{6}{|l|}{ Cor } \\
\hline Não preta & 1435 & 11,6 & 1,00 & & \\
\hline Preta & 257 & 13,2 & 1,14 & 0,$81 ; 1,60$ & \\
\hline \multicolumn{6}{|l|}{ Classe econômica } \\
\hline$A-B$ & 680 & 11,2 & 1,00 & & \multirow{3}{*}{0,52} \\
\hline C & 945 & 12,4 & 1,11 & 0,$84 ; 1,45$ & \\
\hline D-E & 67 & 11,9 & 1,07 & 0,$54 ; 2,12$ & \\
\hline \multicolumn{6}{|l|}{ Peso ao nascer } \\
\hline$<2.500$ & 107 & 13,1 & 1,35 & 0,$75 ; 2,43$ & \multirow{4}{*}{0,82} \\
\hline 2.500-2.999 & 341 & 9,7 & 1,00 & & \\
\hline 3.000-3.999 & 1.144 & 12,7 & 1,31 & 0,$92 ; 1,87$ & \\
\hline$\geq 4.000$ & 100 & 9,0 & 0,93 & 0,$46 ; 1,88$ & \\
\hline \multicolumn{6}{|l|}{ Nascimento } \\
\hline A termo & 1.557 & 11,8 & 1,00 & & \\
\hline Prematuro & 135 & 13,3 & 1,13 & 0,$72 ; 1,78$ & \\
\hline \multicolumn{6}{|l|}{ Prática de atividade física ${ }^{\mathrm{b}}$} \\
\hline Ativo & 849 & 12,2 & 1,00 & & \multirow{3}{*}{0,99} \\
\hline Insuficientemente ativo & 803 & 11,1 & 0,91 & 0,$70 ; 1,18$ & \\
\hline Inativo & 40 & 20,0 & 1,63 & 0,$86 ; 3,11$ & \\
\hline \multicolumn{6}{|c|}{ Tempo gasto com atividades sedentárias ${ }^{\complement}$} \\
\hline$<2$ horas/dia & 302 & 12,3 & 1,05 & 0,$73 ; 1,52$ & \multirow{3}{*}{0,93} \\
\hline 2-3 horas/dia & 635 & 11,7 & 1,00 & & \\
\hline$\geq 4$ horas/dia & 755 & 11,9 & 1,02 & 0,$77 ; 1,37$ & \\
\hline \multicolumn{6}{|l|}{ Estado nutricional } \\
\hline Sem excesso de peso & 1.226 & 10,5 & 1,00 & & \multirow{3}{*}{$<0,01$} \\
\hline Sobrepeso & 311 & 11,3 & 1,07 & 0,$75 ; 1,52$ & \\
\hline Obesidade & 155 & 23,9 & 2,27 & 1,$64 ; 3,14$ & \\
\hline \multicolumn{6}{|l|}{ Perímetro da cintura } \\
\hline $1^{\circ}$ quartil & 430 & 9,5 & 1,00 & & \multirow{4}{*}{0,02} \\
\hline $2^{\circ}$ quartil & 417 & 10,6 & 1,11 & 0,$74 ; 1,66$ & \\
\hline $3^{\circ}$ quartil & 422 & 10,9 & 1,14 & 0,$77 ; 1,70$ & \\
\hline $4^{0}$ quartil & 422 & 16,6 & 1,74 & 1,$21 ; 2,50$ & \\
\hline
\end{tabular}

a Classificação econômica ABEP, 2008; ' ${ }^{\circ}$ Classificação adotada na PeNSE-IBGE, 2009; c Classificação segundo Currie e cols. (2008); ‘Associado ao qui-quadrado da tendência linear.
Tabela 3. Odds ratio ajustada e intervalo de confiança de 95\% (IC 95\%), para os fatores de risco de HAS em adolescentes, 2011

\begin{tabular}{lccc}
\hline & $\begin{array}{c}\text { Odds ratio } \\
\text { ajustada }\end{array}$ & IC (95\%) & p-valor \\
\hline Estado nutricional $^{\mathrm{a}}$ & & & \\
$\quad$ Sem excesso de peso & 1,00 & - & - \\
Sobrepeso & 1,15 & 0,$77 ; 1,71$ & 0,48 \\
$\quad$ Obesidade & 2,72 & 1,$79 ; 4,12$ & $<0,01$ \\
Perímetro da cintura & 1,01 & 0,$99 ; 1,04$ & 0,32 \\
Peso ao nascer & & & \\
$2.500-2.999$ & 1,00 & - & - \\
$\geq 3.000$ & 1,17 & 0,$78 ; 1,75$ & 0,44 \\
$<2.500$ & 1,40 & 0,$71 ; 2,76$ & 0,33 \\
\hline
\end{tabular}

${ }^{a}$ Ajustado por idade, sexo e cor da pele; ' ${ }^{b}$ justado por idade, sexo, cor da pele e IMC.

\section{DISCUSSÃO}

A HAS em adolescentes associou-se à obesidade e linearmente ao perímetro da cintura, mostrando a relevância do excesso de peso como fator de risco para o desfecho avaliado, já nessa fase da vida. $\mathrm{Na}$ análise multivariada, após ajustes, apenas a obesidade permaneceu associada ao desfecho avaliado.

A alta prevalência de HAS encontrada em nosso estudo é retrato do crescente aumento dos níveis tensionais também entre adolescentes no Brasil, semelhante à encontrada em Londrina-PR, cuja prevalência observada foi de 11,8\% (17). Prevalência ainda mais elevada foi encontrada em outro estudo brasileiro, realizado em Recife, que detectou a presença de HAS em 17,3\% dos participantes (18). No estado de Mato Grosso, apenas dois estudos haviam estimado a prevalência de HAS em adolescentes, ambos realizados especificamente na cidade de Cuiabá, sendo os valores encontrados de 2,3\% (19) e 11,2\% (20). Ressalta-se que Borges e cols. (19) avaliaram escolares de 7 a 10 anos, portanto a amostra do estudo contemplou apenas adolescentes de 10 anos de idade. Sendo assim, nossos resultados acrescentam informação importante para o estado de $\mathrm{MT}$, como também para o cenário da HAS em adolescentes no Brasil.

No presente estudo identificou-se importante efeito da obesidade sobre os níveis tensionais dos adolescentes, confirmando a sua participação independente no desenvolvimento da HAS. Segundo a literatura, mais de $75 \%$ dos casos de HAS podem ser atribuídos diretamente à obesidade (21).

Segundo dados recentes da Pesquisa de Orçamento Familiar (POF) 2008-2009 (22), a prevalência de 
excesso de peso na adolescência vem aumentando continuamente em todo o Brasil. Há 30 anos esses valores eram estimados em 3,7\% entre os meninos e 7,6\% entre as meninas. Em 2008/2009 a prevalência foi de 21,7\% e $19,4 \%$ entre meninos e meninas, respectivamente. Esse acréscimo no número de casos pode ser atribuído a interações complexas entre fatores genéticos e ambientais, sendo as alterações nos padrões nutricionais e de atividade física considerados as maiores responsáveis pelo aumento da adiposidade (23).

Dados longitudinais do Estudo do Rio de Janeiro (ERJ) evidenciaram que valores do IMC aumentados associaram-se a maiores valores da PA, outros índices antropométricos e maior prevalência de SM nas três fases da pesquisa, ao longo de 17 anos (24). Engeland e cols. (25) estudaram 128.121 adolescentes que foram acompanhados no mínimo 10 anos depois e observaram que indivíduos adultos que tiveram excesso de peso durante a adolescência apresentam maiores índices de mortalidade quando comparados àqueles com IMC adequado na adolescência, não sendo, no entanto, independente do IMC na idade adulta.

Nossos achados são compatíveis com recentes revisões da literatura $(4,7,21,23)$ que confirmam a hipótese de que a obesidade durante a adolescência é um fator de risco para o desenvolvimento de HAS. Segundo esses autores, essa associação pode acarretar vários efeitos adversos à saúde, em curto e longo prazo, entre eles o desenvolvimento de DCV. No Brasil, esse grupo de doenças tem sido a principal causa de morte e de elevação de custos médico-sociais (12).

A alta correlação entre IMC e perímetro da cintura observada neste estudo $(r=0,88)$ foi semelhante à encontrada por Rosa e cols. (26). Embora a análise bivariada tenha mostrado forte associação entre o maior quartil do perímetro da cintura e a HAS, observou-se que, após ajuste pelo IMC, esse efeito não persistiu, ratificando, dessa forma, a implicação da adiposidade total sobre os níveis tensionais, não confirmando um possível efeito independente da localização abdominal de gordura na ocorrência de HAS, nessa fase da vida.

Nossos resultados contrastam com os obtidos por Lurbe e cols. (27), que mostraram que a distribuição da gordura corporal, estimada pelo perímetro da cintura, é preditor da HAS em adolescentes, independentemente do IMC. Por outro lado, outros autores não confirmaram a necessidade da utilização do perímetro da cintura, além do IMC, como preditor de HAS na adolescência, o que vem ao encontro dos nossos acha- dos com relação à associação entre HAS e localização abdominal de gordura em adolescentes $(28,29)$.

Vale salientar que estudos sobre a avaliação do perímetro da cintura em crianças e adolescentes ainda são escassos, bem como não existem pontos de corte específicos para essa faixa etária na população brasileira (30). Além disso, a identificação de valores adequados de perímetro da cintura é uma tarefa complexa, pois esses são influenciados por sexo, etnia, idade, IMC, entre outros fatores (31).

Assim como o verificado no presente estudo, outras investigações não observaram associação inversa entre HAS e peso ao nascer $(8,32)$, corroborando a hipótese de que a ação de fatores pós-natais exerce maior efeito sobre a PA do que os intrauterinos $(32,33)$.

Neste estudo, outros fatores considerados de risco para a elevação dos níveis tensionais, tais como a inatividade física, a prática de atividades sedentárias, o consumo de álcool e o tabagismo (12), não foram associados à HAS. Outros eventos de ocorrência precoce na vida, como o tabagismo passivo (34), o desmame precoce (35) e o estado nutricional na infância, também não se associaram à HAS.

Entre as limitações do presente estudo, deve-se considerar o seu desenho transversal, que não permite inferir causalidade $(5,17,18)$, embora o critério diagnóstico de HAS preconizado na literatura recomende pelo menos duas medições da PA em momentos diferentes (12). Esse fato poderia acarretar viés na classificação da HAS. Porém, a prevalência de HAS estimada na atual investigação encontra-se próxima àquela observada em outros estudos realizados no Brasil, variando de aproximadamente $2,3 \%$ a $17,3 \%(18,19)$.

Os dados do presente estudo confirmam a obesidade como um fator de risco para o desenvolvimento da HAS entre os adolescentes. Estratégias de saúde pública devem ser instituídas para a prevenção, o controle e o tratamento da HAS e da obesidade na adolescência, a fim de reduzir os prejuízos à saúde em curto e longo prazo.

Agradecimentos: à Coordenação de Aperfeiçoamento de Pessoal de Nível Superior (Capes), processo n ${ }^{\circ}$ 23038.042868/200811; ao Conselho Nacional de Desenvolvimento Científico e Tecnológico (CNPq), processo n 471063/2009-6, e à Fundação de Amparo à Pesquisa de Mato Grosso (Fapemat), processo $n^{\circ}$ $446298 / 2009$.

Declaração: os autores declaram não haver conflitos de interesse científico neste estudo. 


\section{REFERÊNCIAS}

1. Mackay J, Mensah GA. The atlas of heart disease and stroke. World Health Organization; 2004.

2. World Health Organization. World Health Report 2002 - Reducing risks, promoting health life. Geneva; 2002.

3. World Health Organization. Preventing chronic diseases: a vital investment. Geneva; 2005.

4. Falkner B, Lurbe E, Schaefer F. High blood pressure in children: clinical and health policy implications. J Clin Hypertens (Greenwich). 2010;12(4):261-76.

5. Pinto SL, Silva Rde C, Priore SE, Assis AM, Pinto Ede J. [Prevalence of pre-hypertension and arterial hypertension and evaluation of associated factors in children and adolescents in public schools in Salvador, Bahia State, Brazil]. Cad Saude Publica. 2011;27(6):1065-75.

6. National Institutes of Health and Human Services, Lung And Blood Institute. The Fourth Report on Detection, Evaluation and Treatment of High Blood Pressure in Children and Adolescents. Maryland: US Department of Health and Human Services/Public Health Service 2005 Contract No.: (NIH Publication, 05-5267).

7. Flynn JT, Falkner BE. Obesity hypertension in adolescents: epidemiology, evaluation, and management. J Clin Hypertens (Greenwich). 2011;13(5):323-31.

8. Huxley RR, Shiell AW, Law CM. The role of size at birth and postnatal catch-up growth in determining systolic blood pressure: a systematic review of the literature. J Hypertens. 2000;18(7):815-31.

9. Gonçalves-Silva RM, Valente JG, Lemos-Santos MG, Sichieri R. [Household smoking and stunting for children under five years]. Cad Saude Publica. 2005;21(5):1540-9.

10. Goncalves-Silva RM, Sichieri R, Ferreira MG, Pereira RA, Muraro AP, Moreira NF, et al. [The school census as a search strategy for children and adolescents in epidemiological studies]. Cad Saude Publica. 2012;28(2):400-4.

11. Christofaro DG, Fernandes RA, Gerage AM, Alves MJ, Polito MD, Oliveira AR. Validation of the Omron HEM 742 blood pressure monitoring device in adolescents. Arq Bras Cardiol. 2009;92(1):10-5.

12. Sociedade Brasileira de Cardiologia, Sociedade Brasileira de Hipertensão, Sociedade Brasileira de Nefrologia. VI Diretrizes Brasileiras de Hipertensão. Arq Bras Cardiol. 2010;95(1 supl.1):1-51.

13. Onis M, Onyango AW, Borghi E, Siyam A, Nishida C, Siekmann J. Development of aWHO growth reference for school-aged children and adolescents. Bull World Health Organ. 2007;85(9):660-7.

14. ABEP-Associação Brasileira de Empresas de Pesquisas. Códigos e guias: CCEB - Critério de Classificação Econômica Brasil. São Paulo: ABEP; 2008.

15. IBGE - Instituto Brasileiro de Geografia e Estatística. Pesquisa Nacional de Saúde do Escolar. Rio de Janeiro: IBGE; 2009.

16. Currie C, Gabhainn SN, Godeau E, Roberts C, Smith R, Currie D, et al. Inequalities in young people's health. HBSC international report from the 2005/2006 survey. Copenhagen: WHO Regional Office for Europe; 2008.

17. Christofaro DG, Ritti-Dias RM, Fernandes RA, Polito MD, Andrade SM, Cardoso JR, et al. High blood pressure detection in adolescents by clustering overall and abdominal adiposity markers. Arq Bras Cardiol. 2011;96(6):465-70.

18. Gomes BM, Alves JG. [Prevalence of high blood pressure and associated factors in students from public schools in Greater
Metropolitan Recife, Pernambuco State, Brazil, 2006]. Cad Saude Publica. 2009;25(2):375-81.

19. Borges LM, Peres MA, Horta BL. [Prevalence of high blood pressure among schoolchildren in Cuiaba, Midwestern Brazil]. Rev Saude Publica. 2007;41(4):530-8.

20. Vieira MA, Carmona DPD, Anjos LA, Souza T, Espinosa MM, Ribeiro RLR. Pressão arterial de crianças e adolescentes de escolas públicas de Cuiabá, Mato Grosso. 2009;22:473-5.

21. Ho TF. Cardiovascular risks associated with obesity in children and adolescents. Ann Acad Med Singapore. 2009;38(1):48-9.

22. IBGE Instituto Brasileiro de Geografia e Estatística. Pesquisa de Orçamentos Familiares - 2008-2009: Antropometria de crianças, adolescentes e adultos. Rio de Janeiro: IBGE; 2010.

23. Biro FM, Wien M. Childhood obesity and adult morbidities. Am J Clin Nutr. 2010;91(5):1499S-505S.

24. Fonseca FL, Brandao AA, Pozzan R, Campana EM, Pizzi OL, Magalhaes $\mathrm{ME}$, et al. [Overweight and cardiovascular risk among young adults followed-up for 17 years: the Rio de Janeiro study, Brazil]. Arq Bras Cardiol. 2010;94(2):193-201, 7-15, 196-204.

25. Engeland A, Bjorge T, Tverdal A, Sogaard AJ. Obesity in adolescence and adulthood and the risk of adult mortality. Epidemiology. 2004;15(1):79-85.

26. Rosa ML, Mesquita ET, da Rocha ER, Fonseca Vde M. Body mass index and waist circumference as markers of arterial hypertension in adolescents. Arq Bras Cardiol. 2007;88(5):573-8.

27. Lurbe E, Alvarez V, Redon J. Obesity, body fat distribution, and ambulatory blood pressure in children and adolescents. J Clin Hypertens (Greenwich). 2001;3(6):362-7.

28. Kuschnir MC, Mendonca GA. Risk factors associated with arterial hypertension in adolescents. J Pediatr (Rio J). 2007;83(4):335-42.

29. Ribeiro RC, Lamounier JA, Oliveira RG, Bensenor IM, Lotufo PA. Measurements of adiposity and high blood pressure among children and adolescents living in Belo Horizonte. Cardiol Young. 2009;19(5):436-40.

30. Pinto IC, Arruda IK, Diniz Ada S, Cavalcanti AM. [Prevalence of overweight and abdominal obesity according to anthropometric parameters and the association with sexual maturation in adolescent schoolchildren]. Cad Saude Publica. 2010;26(9):1727-37.

31. Klein S, Allison DB, Heymsfield SB, Kelley DE, Leibel RL, Nonas C, et al. Waist circumference and cardiometabolic risk: a consensus statement from Shaping America's Health: Association for Weight Management and Obesity Prevention; NAASO, The Obesity Society; the American Society for Nutrition; and the American Diabetes Association. Am J Clin Nutr. 2007;85(5):1197-202.

32. Falkner B, Hulman S, Kushner H. Effect of birth weight on blood pressure and body size in early adolescence. Hypertension. 2004;43(2):203-7.

33. Taylor SJ, Whincup PH, Cook DG, Papacosta O, Walker M. Size at birth and blood pressure: cross sectional study in 8-11 year old children. BMJ. 1997;314(7079):475-80.

34. Araujo TL, de Lopes MV, Cavalcante TF, Guedes NG, Moreira $\mathrm{RP}$, Chaves ES, et al. [Analysis of risk indicators for the arterial hypertension in children and teenagers]. Rev Esc Enferm USP. 2008;42(1):120-6.

35. Martin RM, Davey Smith G, Mangtani P, Tilling K, Frankel S, Gunnell D. Breastfeeding and cardiovascular mortality: the Boyd Orr cohort and a systematic review with meta-analysis. Eur Heart J. 2004;25(9):778-86. 